\title{
Urine Composition in Pigs is Regulated in Both the Ureter and the Bladder
}

\author{
L. K. JAKOBSEN ${ }^{1,2}$, K.-E. ANDERSSON ${ }^{1,3}$, R. NØRREGAARD ${ }^{1}$, L. H. OLSEN ${ }^{1,2}$ \\ ${ }^{1}$ Department of Clinical Medicine, Aarhus University, Denmark, ${ }^{2}$ Department of Urology, Aarhus \\ University Hospital, Denmark, ${ }^{3}$ Department of Gynecology and Obstetrics, Aarhus University \\ Hospital, Denmark
}

Received November 19, 2018

Accepted May 20, 2019

Epub Ahead of Print August 19, 2019

\section{Summary}

The former perception of the urothelium as an impermeable barrier has been revised during the last decade, as increasing evidence of changes in urine composition during its passage of the urinary tract has been presented. Since differences in urothelial permeability between upper and lower urinary tract have been found, our aim is to demonstrate whether changes in urine composition occur during passage through the ureter. We studied consecutive urine samples from both renal pelvises in six pigs and compared them to samples from the bladder and distal ureter. We further sampled urine during storage in the bladder at a fixed volume. All samples were analysed by measuring osmolality and $\mathrm{pH}$, along with the concentration of the following parameters: $\mathrm{Na}^{+}, \mathrm{K}^{+}, \mathrm{Cl}^{-}$, creatinine, urea. Urine alkalinity increased significantly during passage of the ureter. Creatinine concentration, $\mathrm{pH}$ and $\mathrm{K}^{+}$increased significantly during the passage from pelvis to the bladder. All other parameters increased non-significantly during the passage to the bladder. The increase in concentration was more pronounced at low concentrations in the pelvis. During storage in the bladder, there was a significant increase in urea concentration. Changes in the composition of urine occur during its passage from the renal pelvis to the bladder and during storage in the bladder. Despite the brief transit time, significant changes in alkalinity were found already during passage through the ureter.

\section{Key words}

Urine composition • Animal model

\section{Corresponding author}

L. K. Jakobsen, Department of Clinical Medicine, Aarhus
University, Palle Juul-Jensens Boulevard 99, 8200 Aarhus N, Denmark. E-mail: lottekj@clin.au.dk

\section{Introduction}

During the last decade increasing attention has been given to the various functions of the urothelium (Khandelwal et al. 2009, Birder and Andersson 2013, Lasič et al. 2015). Tight junctions between urothelial cells and lining of the luminal urothelial surface by uroplakins and glycans work together in reducing urothelial permeability (Riedel et al. 2005). This accounts for the historical perception of the urothelium acting as the "skin" of the urinary tract allowing nothing to pass (Hicks et al. 1974, Lewis 2000). However, water channels (aquaporins) and several other transport proteins have been identified in mammalian urothelium, and probably the alleged barrier is not always complete (Rubenwolf et al. 2009, Spector et al. 2002, Spector et al. 2011, Spector et al. 2013).

Currently, the accepted dogma is that urothelial permeability is consistent throughout the urinary tract. This is largely based on the assumption that morphologically the urothelium lining the ureter, bladder and urethra are the same. However, their embryological derivation is quite different, urothelium of the renal pelvis and the ureter being of mesodermal origin, while the lining of the bladder and urethra is of endodermal origin (Al-Kurdi 2017). There is also evidence suggesting that despite apparent histological homology, protein expression on the surface of urothelial umbrella cells is 
not consistent (Riedel et al. 2005, Williams et al. 2016) showed differences in urothelial permeability between the upper urinary tract and the bladder.

Many factors, such as aquaporins and urea transporters, have been shown to be involved in the regulation of the urothelial lining, including its permeability normally and in different disorders (Kreft et al. 2009, Al-Kurdi 2017). For example, conditions such as partial bladder outlet obstruction, dehydration and urothelial carcinoma has been shown to affect aquaporin expression in the bladder (Rubenwolf et al. 2009, Spector et al. 2002, Kim et al. 2010, Rubenwolf et al. 2012). Previous studies on the American black bear indicated reabsorption of urine from the bladder, probably an advantageous ability during hibernation to prevent dehydration and improve hygiene (Nelson et al. 1973a, Nelson et al. 1973b, Spector et al. 2015). Most studies on changes in urine composition have focused on the storage period in the bladder, but although the transit time in the ureter is brief, the contact surface between urothelium and urine is considerable and a possible change might occur also in the ureter. Even though this has been addressed previously in human patients who had undergone treatment for urinary tract stones, or surgery including bilateral cutaneous ureterostomy (Cahill et al. 2003, Shafik et al. 2005), the issue has not been clarified. So, we want to further explore the possibility that the ureter has an important role in the regulation of urine composition under normal conditions. Since the pig is often used as a model in translational urological research, we employed a pig model to examine the changes of urine composition occurring when urine is passing from the renal pelvis to the bladder. In particular, we wanted to assess whether concentration changes primarily occur during the passage through the ureter, after entering the bladder, or during storage in the bladder. Such changes in concentration could be relevant when designing new treatments or diagnostic tests. The findings may have implications both regarding bladder dysfunction, but also in urinary stone disease.

\section{Material and Methods}

\section{Experimental animals and anesthesia}

Ethical approval of experiments was granted by the Danish Animal Experiments Inspectorate (Approval no: 2016-15-0201-00942). All procedures were carried out at Aarhus University and conformed to the Danish National Guidelines for care and handling of animals.
Female pigs $(\sim 40 \mathrm{~kg})$ crossbred Landrace/ Yorkshire/Duroc were obtained from a commercial source and kept at the farming facilities of Aarhus University at least one week prior to experiments, assuring health conditions. They were fasted over night with free access to water, and weighed at the day of the experiment. Anesthesia was induced with intravenous injection of hypnomidate $(0.5 \mathrm{mg} / \mathrm{kg})$ and maintained with sevoflurane $(\sim 1.3 \mathrm{MAC})$ and intravenous infusion of fentanyl $(12.5 \mu \mathrm{g} / \mathrm{kg} / \mathrm{h})$. Animals were intubated and ventilated automatically throughout the duration of the experiment, with continuous monitoring of temperature and vital parameters. All pigs were overhydrated following a standardized protocol during the experiment with intravenous infusion of equal volumes of isotonic sodium chloride and Ringer acetate (in total $\sim 1,000 \mathrm{ml} / \mathrm{h}$ ), based on experience with low diuresis in anaesthetised pigs. Upon finishing the experiments animals were euthanized by intravenous injection of pentobarbital $(40 \mathrm{mg} / \mathrm{kg})$ while still anesthetised.

\section{Surgical procedures}

Intravenous catheters were placed in superficial veins of both ears for anesthesia and IV fluids. The left carotid artery was surgically exposed, and a vascular sheath was placed to monitor blood pressure and for drawing blood samples. A transurethral catheter (Rüsch, silicone, ch. 14, Teleflex Medical, Athlone, Ireland) was placed in the bladder. The renal pelvis on both sides was accessed retroperitoneally through small transverse flank incisions, and a pig-tail catheter (M-drain, $7 \mathrm{Fr}$, Mermaid medical, Copenhagen, Denmark) was inserted directly into the pelvis by Seldinger technique, and suture fixed. Afterwards a Pfannenstiel incision was made and the distal left ureter was exposed and cut at the entrance to the bladder. The bladder site was ligated. An 8 Fr babyfeeding tube (Unomedical, Lejre, Denmark) was inserted $2-3 \mathrm{~cm}$ into the distal left ureter and held in place with a suture.

\section{Collecting and handling samples}

The pig was placed in prone position and the four urinary catheters were each connected to a tap and placed in collecting tubes (Nunc Cryotube Vials, Thermo Scientific, Massachusetts, USA). Ten sets (forty samples) of simultaneously collected paired urine samples of $4 \mathrm{ml}$ from the four collection sites were obtained from each pig. Experimental setting is depicted in Figure 1. 


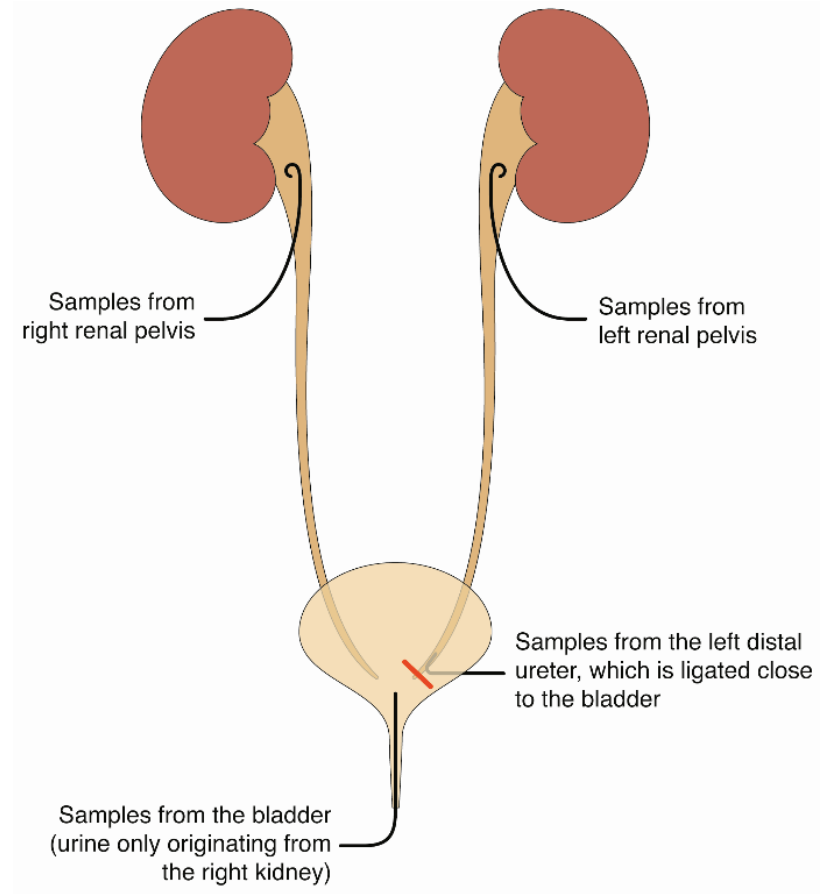

Fig. 1. Experimental setting. To evaluate changes from the renal pelvis to the bladder, samples are taken from these locations on the right side. On the left side, samples are taken from the renal pelvis to the distal ureter, and the entry point of the left ureter to the bladder is closed, to avoid interference with the right side sample sets.

Afterwards the bladder catheter was clamped and the bladder filled. If diuresis from the right side was insufficient, urine from the left side catheters was added. When reaching a bladder volume of $300 \mathrm{ml}$, confirmed by aspiration, the right ureter was ligated, leaving the bladder with a constant volume. Five additional samples of bladder urine were now collected with 30-minute intervals to evaluate concentration changes during urine storage in the bladder.

In order to exclude significant systemic changes during the experiment, a total of five blood samples from each pig were drawn from the central arterial catheter during the entire experiment, to evaluate serum concentrations of the examined parameters $\left(\mathrm{Na}^{+}, \mathrm{K}^{+}, \mathrm{Cl}^{-}\right.$, creatinine, urea and osmolality).

Each urine sample was immediately separated into 2 tubes and the $\mathrm{pH}$ was measured using an InLab ${ }^{\circledR}$ Semi-Micro electrode, connected to a SevenCompact $^{\mathrm{TM}} \quad$ pH-meter (Mettler Toledo, Hasselager, Denmark), automatically correcting for temperature. Hereafter both samples were snap frozen in liquid nitrogen and stored at $-80{ }^{\circ} \mathrm{C}$ until further analysis. Osmolality was determined using the OSMOMAT ${ }^{\circledR} 030$ cryoscopic osmometer (Gonotec, Berlin, Germany).
All other parameters were analysed in the Advia ${ }^{\circledR} 1800$ (Siemens Healthineers, Erlangen, Germany) according to standard procedures. Creatinine and urea was measured by spectrophotometry, with intra- and inter-assay precisions below 3 and $4 \mathrm{CV} \%$, respectively. Sodium, potassium and chloride were determined by ion selective electrodes, with intra- and inter-assay precisions below 2.7 and $3.7 \mathrm{CV} \%$, respectively. After separation blood samples were centrifuged at $3,000 \mathrm{rpm}$ for $10 \mathrm{~min}$ at $4{ }^{\circ} \mathrm{C}$. Serum was snap frozen and stored at $-80{ }^{\circ} \mathrm{C}$ until further analysis, which was done following same procedure as with the urine samples.

\section{Statistical analysis}

Statistical analysis was performed in Graphpad Prism 7.0a (GraphPad Software Inc., La Jolla, CA, USA). Results from $\mathrm{pH}$-measurements were converted into hydrogen-ion concentrations $\left(H^{+}=10^{-p H}\right)$ for accurate statistical processing. The relative concentration changes of all parameters were compared in pairs of upper sample (renal pelvis) and lower sample (bladder or distal ureter) with a ratio paired $t$-test. The mean ratios were then combined for all pigs, and analysed by one-sample $t$-test against 1. Assumptions for validity of testing were checked graphically with qq-plots, correlation scatter and Bland-Altman-plots. Linear regression was performed to evaluate changes over time, analyzing paired sample ratios, serum samples and the five bladder urine samples from each pig, collected during urine storage at fixed volume.

\section{Results}

Six female pigs with a mean weight of $39.1 \mathrm{~kg}$ (range: $37.3-40 \mathrm{~kg}$ ) were included in the study. Sixty sample sets were available to compare renal pelvis and bladder urine. For comparison of renal pelvis to distal ureter urine, 48 sample sets were available, since twelve sets had to be excluded due to technical issues such as haematuria or catheter malfunction (ten of the excluded samples were from one pig). Thirty serum samples were available for osmolality, and 25 available for testing other parameters. The latter numbers were the same regarding samples from prolonged storage in the bladder, where also 30 samples were available for $\mathrm{pH}$ measuring.

Urine alkalinity showed a marked and uniform increase on both sides, with a significant increase in $\mathrm{pH}$ from pelvis to bladder (mean decrease in $\mathrm{H}^{+}$: $32 \% \mathrm{CI}$ : $20-45 \%$ ) and from pelvis to distal ureter (mean $\mathrm{H}^{+}$ 
decrease: $23 \% \mathrm{CI}: 14-31 \%$ ), corresponding to a mean increase of $0.17(0.10-0.26) \mathrm{pH}$ points between pelvis and bladder, and $0.11(0.07-0.16) \mathrm{pH}$ points from pelvis to distal ureter (Fig. 2).

Concentration of all other parameters $\left(\mathrm{Na}^{+}, \mathrm{K}^{+}\right.$, $\mathrm{Cl}^{-}$, creatinine, urea and osmolality), showed a tendency to increase in the sample sets from the renal pelvis and the bladder. However, apart from the $\mathrm{pH}$, these changes were statistically significant only regarding creatinine and $\mathrm{K}^{+}$(Table 1). Creatinine showed a mean increase of $17 \%$ (CI: $2-32 \%$ ) and $\mathrm{K}^{+}$a mean increase of $12 \%$ (CI: $1-23 \%$ ). Evaluating the sample sets comparing the renal pelvis and the distal ureter did not show consistent results on any parameters, apart from the $\mathrm{pH}$. We did not observe any other changes that were statistically significant in these sample sets (Table 1).

The direction of concentration changes regarding all parameters was not uniform, but showed some sample pairs with increasing concentrations and some with decreases, as illustrated in Figure 3a, b. Generally, a low concentration in the pelvis would warrant a prominent increase in concentration, whereas a high concentration in the pelvis would decrease or remain unchanged in the lower sample (Fig. 3a, b). In some instances, this tendency was extremely marked, as illustrated in Figure $3 \mathrm{c}$, and this effect gives rise to the tendency of urea to decrease through the ureter, a finding mainly based on sample sets with a high concentration in the pelvis.

The final part of the experiment, evaluating concentration changes during storage in the bladder with a fixed volume, showed increasing concentration of urea in all pigs (Fig. 4). Regarding all other parameters $\left(\mathrm{Na}^{+}\right.$, $\mathrm{K}^{+}, \mathrm{Cl}^{-}, \mathrm{pH}$, creatinine and osmolality) results were diverging between pigs, with some pigs showing decreases and other pigs increases in concentration during storage. Considering all pigs together urea concentration showed a mean increase of $14 \%$ (CI: $1-27 \%$ ), all other parameters showed no significant changes.

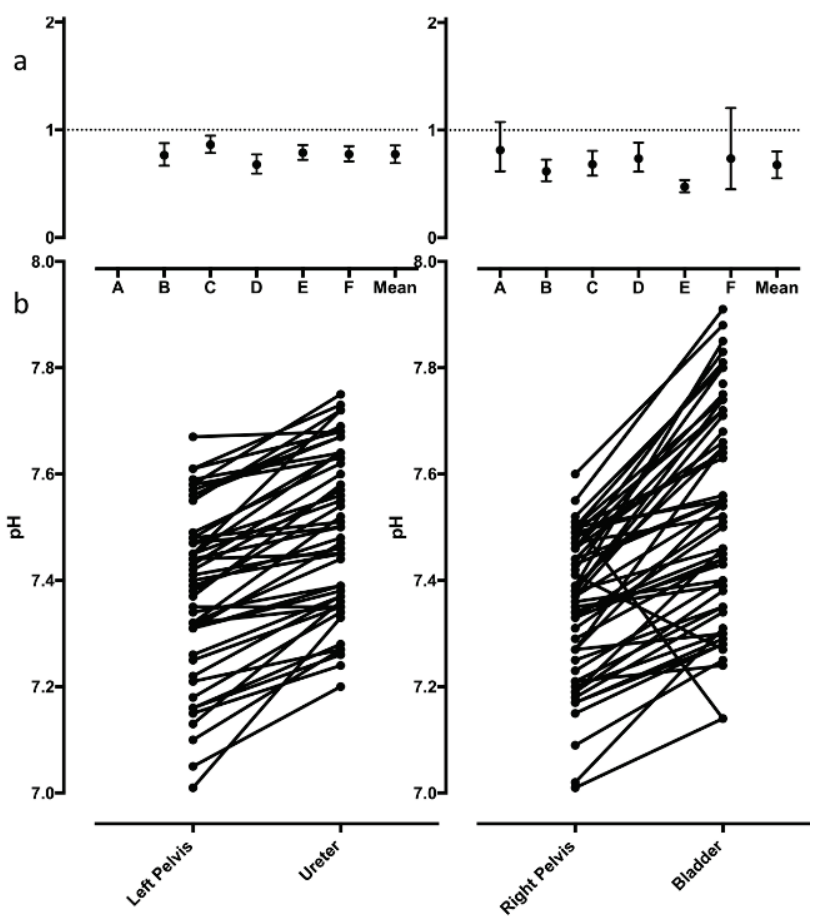

Fig. 2. Changes in $\mathrm{pH}$ from renal pelvis to either distal ureter or bladder. (a) Mean relative changes in separate pigs (A-F) and the overall mean relative change (Mean). Samples comparing renal pelvis to distal ureter on the left, samples comparing renal pelvis to the bladder on the right. (Error bars: $95 \%$ confidence intervals, dotted line: no change).

$$
y=\frac{H^{+} \text {concentration lower sample (bladder or distal ureter) }}{H^{+} \text {concentration renal pelvis }}
$$

(b) Plots of $\mathrm{pH}$ changes in paired samples from both sides. Sample location indicated on the $\mathrm{x}$-axis. $\mathrm{pH}$ increases in most sample pairs.

Table 1. Mean relative changes in concentration (95\% confidence intervals).

\begin{tabular}{|c|c|c|c|c|}
\hline & \multicolumn{4}{|c|}{ Relative changes } \\
\hline & Pelvis to bladder & $\mathbf{p}$ & Pelvis to distal ureter & $\mathbf{p}$ \\
\hline Osmolality & $1.06(1.00-1.11)$ & 0.06 & $1.01(0.91-1.11)$ & 0.81 \\
\hline$K+$ & $1.12(1.01-1.23)$ & 0.04 & $0.96(0.73-1.19)$ & 0.65 \\
\hline $\mathrm{Na}+$ & $1.03(0.98-1.07)$ & 0.16 & $1.03(0.97-1.10)$ & 0.25 \\
\hline $\mathrm{Cl}-$ & $1.04(0.98-1.09)$ & 0.14 & $1.02(0.94-1.09)$ & 0.60 \\
\hline Creatinine & $1.17(1.02-1.32)$ & 0.03 & $0.97(0.72-1.21)$ & 0.71 \\
\hline Urea & $1.15(0.96-1.33)$ & 0.09 & $0.87(0.70-1.04)$ & 0.10 \\
\hline$H^{+}\left(10^{-p H}\right)$ & $0.68(0.55-0.80)$ & $<0.01$ & $0.77(0.69-0.86)$ & $<0.01$ \\
\hline
\end{tabular}

Compared as Lower/Upper ratio - Lower $=$ Bladder or distal ureter, Upper $=$ Renal pelvis. Significant changes are marked by colored background. $\mathrm{p}$-values $<0.05$ reject the hypothesis of ratio $=1$. 


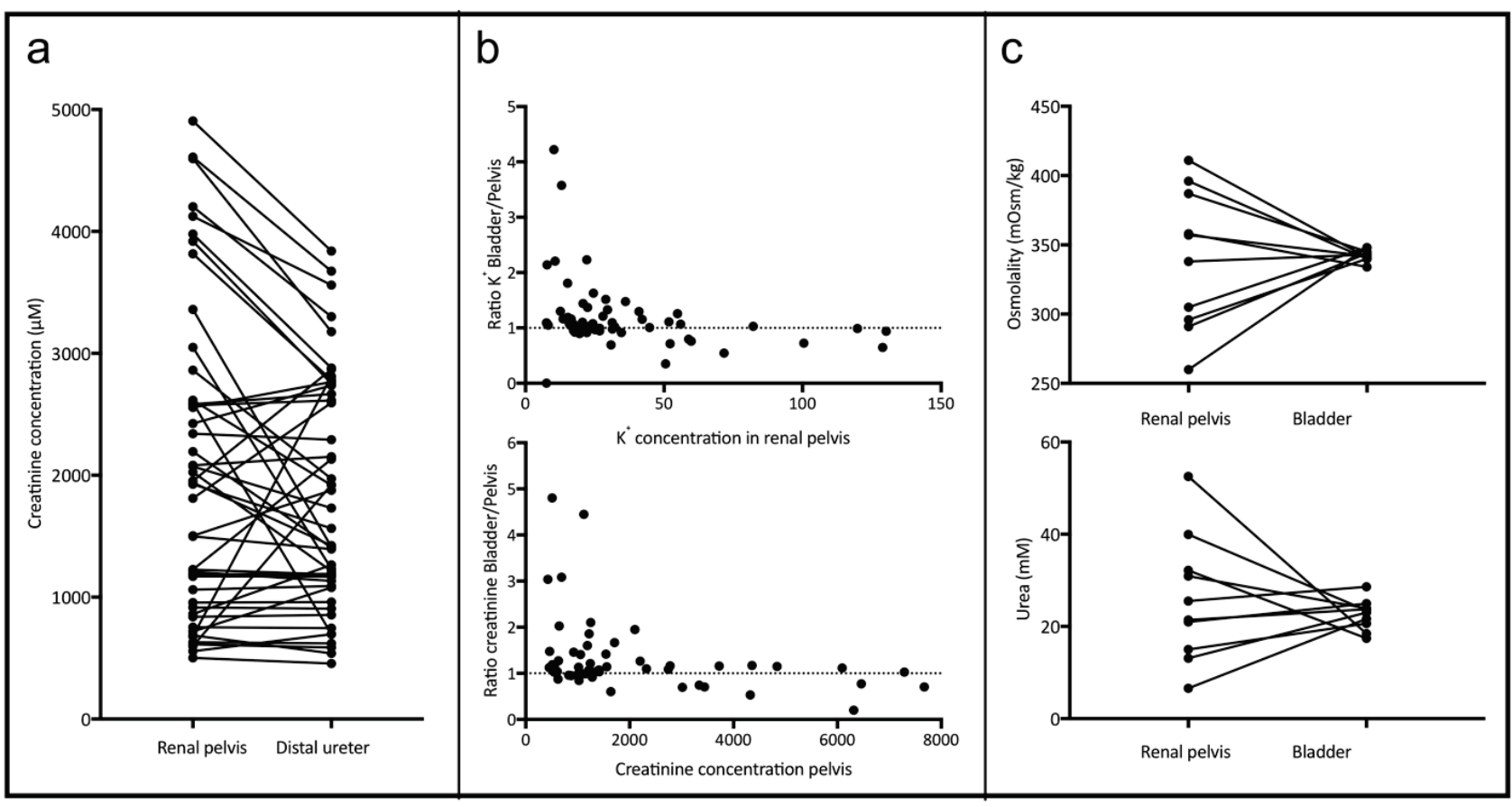

Fig. 3. (a) Plots of creatinine concentration in paired samples evaluating changes from the renal pelvis to the distal ureter. Direction of changes is not uniform between sample sets. In general, when starting at a high concentration there will be a decrease towards the lower sample, and vice versa, as further illustrated in panel b. (b) The relative changes in concentration of $\mathrm{K}^{+}$(top) and creatinine (bottom) plotted as a function of the concentration in the renal pelvis. Relative increases are greater when the renal pelvis concentration is low. (c) Changes of osmolality and urea concentration from one pig showing a marked example of the above described effect.

Relative concentration changes between samples from the renal pelvis and samples from the bladder or distal ureter were overall not affected by duration of anesthesia as evaluated graphically and by linear regression (data not shown).

Serum concentrations of the examined parameters were overall stable throughout the experiment regarding most parameters. There was, however, an overall mean decrease of $2.3 \%$ (CI: $0.7-4.0 \%$ ) per hour in serum creatinine as estimated by linear regression $(\mathrm{p}=0.02)$.

\section{Discussion}

We found an increased urine alkalinity during passage of the ureter and a slightly larger increase after entry of urine into the bladder. However, no further significant changes in $\mathrm{pH}$ occurred after prolonged storage of urine in the bladder. This could indicate that the reduction in $\mathrm{H}^{+}$concentration is predominantly occurring in the ureter. Concentrations of creatinine and $\mathrm{K}^{+}$increased significantly from the renal pelvis to the bladder. During prolonged storage in the bladder we observed an increasing urea concentration.

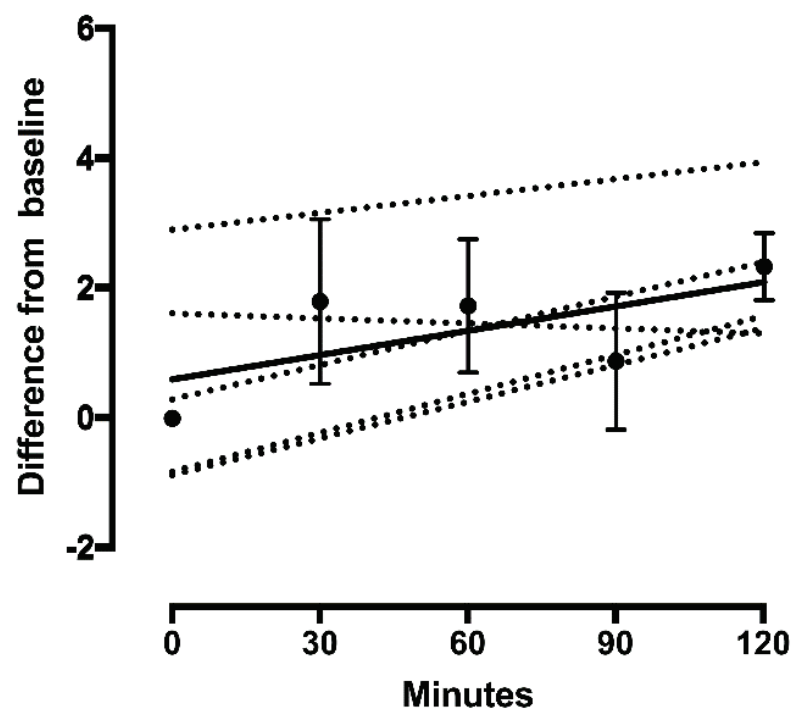

Fig. 4. Change of urea concentration during two hours of storage in the bladder. On the $y$-axis is indicated the absolute change from baseline concentration. The dotted lines represent fitted linear regression curves from individual pigs. The solid line is the overall curve-fit. Dots and error-bars represent mean values and SEM.

Our findings are consistent with those shown in human subjects by Cahill et al. (2003). Their studies were performed on mainly unilateral samples from patients with renal stones, comparing concentrations in the 
operated renal pelvis to concentrations in bladder samples. They further had a few sample sets from both renal pelvises, and found no significant differences between the two sides. In addition, they found higher $\mathrm{pH}$, $\mathrm{Na}^{+}, \mathrm{K}^{+}$and osmolality in bladder samples compared to renal pelvis samples, and furthermore observed (the same effect as we did) that the direction and degree of concentration changes depends on the primary concentration. However, they were not able to distinguish between changes occurring in the ureter and those occurring in the bladder.

With our animal model design, we can speculate that since the $\mathrm{pH}$ increased significantly in the sample pairs comparing distal ureter to the renal pelvis, at least part of the change must occur in the ureter. Shafik and colleagues (Shafik et al. 2005) conducted a series of studies, all examining urine sample pairs from human subjects. In one study, they examined concentration changes occurring in the urethra by sampling voided and vesical urine. Surprisingly, they found increases in both $\mathrm{pH}, \mathrm{Na}^{+}, \mathrm{K}^{+}$and osmolality, which they attributed to either the interaction between urine and urothelium in the urethra or alternatively to the addition of mucus from prostatic and urethral glands (Shafik et al. 2004). In another study Shafik et al. (2006) compared samples from the right renal pelvis to samples from the bladder, disregarding the contribution from the left kidney. They observed increases in all examined parameters; $\mathrm{pH}$, osmolality, $\mathrm{Na}^{+}$and $\mathrm{K}^{+}$. In a further study they focused on changes occurring through the passage of the ureter (Shafik et al. 2005), and compared urine samples from the renal pelvis with samples from ureterocutaneostomies in patients who had previously undergone cystectomy. They concluded that concentrations of $\mathrm{Na}^{+}$ and $\mathrm{K}^{+}$increase, and that only insignificant increases in $\mathrm{pH}$ and osmolality occurred. Contributing to the differences in results between our study and that of Shafik et al. could be that urinary $\mathrm{pH}$ is different and that they studied patients, whereas we studied normal pigs. Their human subjects had a urinary $\mathrm{pH} \sim 6$ as compared to our pig subjects with $\mathrm{pH} \sim 7.3$. We demonstrated a mean decrease in $\mathrm{H}^{+}$concentration of $23 \%$ through the ureter. The larger change we observed in the sample sets, where the urine had passed the ureter as well as the bladder (32\%) suggest that there is a further change occurring in the bladder. However, the results after prolonged storage in the bladder where urine alkalinity does not change significantly contrarily indicate that the alkalinisation primarily happens in the ureter. The transit time through the ureter is short, but the contact surface between urine and urothelium is much larger than in the bladder, justifying the assumption of changes occurring predominantly in the ureter. As mentioned in the introduction, there may be differences in permeability between the urothelium in the upper tract compared to that in the bladder. However, the baseline $\mathrm{pH}$ of the bladder urine was significantly higher than $\mathrm{pH}$ of renal pelvis urine, and this might also explain the lack of significant change during prolonged storage. These $\mathrm{pH}$ changes might be especially relevant in patients suffering from urinary stone disease, as $\mathrm{pH}$ influences the likeliness of stone formation. It is relevant to know that a urine sample from the bladder might not reflect the $\mathrm{pH}$ found in the pelvis, where the stones are usually formed.

Thus, the findings in our present study are in concordance with previous studies regarding the direction of concentration changes, but we find diverging answers to the question whether the $\mathrm{pH}$ is mainly affected by the passage of the ureter or by the storage in the bladder. Rubenwolf et al. (2012) developed a cultured differentiated cell layer of human urothelial cells and demonstrated permeability to radioactively-labelled water and urea, with an increased flux after establishing an osmotic gradient. They further demonstrated expression of AQP3, 4, 7 and 9, and found upregulation of AQP3 after exposure to hyper-osmotic $\mathrm{NaCl}$ and downregulation caused by hypo-osmotic $\mathrm{NaCl}$. AQPs were then blocked with $\mathrm{HgCl}_{2}$ and permeability re-evaluated. Permeability to both water and urea was reduced following AQP-blockage by $\mathrm{HgCl}_{2}$ in a concentration dependent manner, indicating that the permeability might be mediated by AQPs. In a previous study, also by Rubenwolf et al. (2009), expression of AQP3, 4, 7, 9, and 11 transcripts was demonstrated in samples from human ureter. In a recent study we demonstrated expression of AQP1, 3, 9, and 11 transcripts in the fetal porcine ureter (Jakobsen et al. 2018). The increases in concentration of all evaluated parameters (except for $\mathrm{H}^{+}$, which decreases) would correspond well with active reabsorption of water from the urinary tract. The regulation of urinary acidification has been well studied in the kidney, and the main processes involved in the maintenance of systemic acidbase homeostasis are reabsorption of filtered $\mathrm{HCO}_{3}$ and excretion of acid and ammonium. A variety of acid-base transporters are involved in the processes of urinary $\mathrm{pH}$ regulation. The collecting ducts are considered the final site for urine acid-base regulation, but our findings 
suggest that the regulation continues at least through the ureter. Embryologically, both the ureters and the collecting ducts originate from the ureteric bud. With that in mind, similarities in their function seems plausible. A urinary acidification defect caused by diminished net $\mathrm{H}^{+}$secretion and/or $\mathrm{HCO}_{3}$-reabsorption has been shown following urinary tract obstruction (Wang et al. 2008). However, obstruction also affects the ureters, and it cannot be excluded that some of these transporters are located also in the urothelium of the ureters and play a role in the changes in $\mathrm{H}^{+}$concentration occurring in the normal situation. Obviously, this possibility could motivate further study. To increase diuresis in our model, all pigs were overhydrated with intravenous infusion of equal volumes of isotonic sodium chloride and ringer acetate during the experiment. Spector et al. (2011) and Spector et al. (2013) studied how urothelial transport of water and solutes may occur and may be regulated physiologically by animal hydration status. They instilled urine collected during 2-day water deprivation, water loading, or ad libitum water intake into isolated in situ bladder(s) of groups of rats undergoing one of the same three hydration states. They retrieved the urine and measured changes in urine volume and concentrations and quantities of urine urea nitrogen, creatinine, and other solutes. For each ionic species, two factors independently influenced transport: Instilled urinary ion concentration and animal hydration state. To what extent the hydration state may have influenced our results can only be speculated. Since all pigs were subjected to the same degree of hydration, qualitative differences would not be expected. On the other hand, compared to normal, quantitative differences cannot be excluded.

In conclusion, this study has shown that changes in urine composition occur when urine passes from the renal pelvis to the bladder and during storage in the bladder. Our results suggest that some of the changes occur already during the passage through the ureter. The transport mechanisms involved and the implications of the findings motivate further study.

\section{Conflict of Interest}

There is no conflict of interest.

\section{Acknowledgements}

Thanks to medical student Ida Faurschou and Dr. Amr Kamal Salama for assisting with the experiments. Thanks to professor Jens Christian Djurhuus, professor Christopher Fry and senior lecturer Bahareh Vahabi for discussions on the study protocol. Thanks to Mettler Toledo for lending the $\mathrm{pH}$-meter. Thanks to Mermaid Medical for supplying pig-tail catheters. Thanks to senior scientist Torben Larsen and laboratory technician Gitte Kall for assistance with sample analysis.

\section{References}

AL-KURDI B: Hierarchical transcriptional profile of urothelial cells development and differentiation. Differentiation 95: 10-20, 2017.

BIRDER L, ANDERSSON KE: Urothelial signaling. Physiol Rev 93: 653-680, 2013.

CAHILL DJ, FRY CH, FOXALL PJ: Variation in urine composition in the human urinary tract: Evidence of urothelial function in situ? J Urol 169: 871-874, 2003.

HICKS RM, KETTERER B, WARREN RC: The ultrastructure and chemistry of the luminal plasma membrane of the mammalian urinary bladder: a structure with low permeability to water and ions. Philos Trans $R$ Soc Lond B Biol Sci 268: 23-38, 1974.

JAKOBSEN LK, TRELBORG KF, KINGO PS, HOYER S, ANDERSSON KE, DJURHUUS JC, NORREGAARD R, OLSEN LH: Aquaporin expression in the fetal porcine urinary tract changes during gestation. Physiol Res 67: 283-292, 2018.

KHANDELWAL P, ABRAHAM SN, APODACA G: Cell biology and physiology of the uroepithelium. Am J Physiol Renal Physiol 297: F1477-F1501, 2009.

KIM SO, SONG SH, AHN K, KWON D, PARK K, RYU SB: Changes in aquaporin 1 expression in rat urinary bladder after partial bladder outlet obstruction: preliminary report. Korean J Urol 51: 281-286, 2010.

KREFT ME, JEZERNIK K, KREFT M, ROMIH R: Apical plasma membrane traffic in superficial cells of bladder urothelium. Ann N Y Acad Sci 1152: 18-29, 2009.

LASIČ E, VIŠNJAR T, KREFT ME: Properties of the urothelium that establish the blood-urine barrier and their implications for drug delivery. Rev Physiol Biochem Pharmacol 168: 1-29, 2015. 
LEWIS SA: Everything you wanted to know about the bladder epithelium but were afraid to ask. Am J Physiol 278: F867-F874, 2000.

NELSON RA, WAHNER HW, JONES JD, ELLEFSON RD, ZOLLMAN PE: Metabolism of bears before, during, and after winter sleep. Am J Physiol 224: 491-496, 1973a.

NELSON RA: Winter sleep in the black bear. A physiologic and metabolic marvel. Mayo Clinic Proc 48: 733-737, 1973b.

RIEDEL I, LIANG FX, DENG FM, TU L, KREIBICH G, WU XR, SUN TT, HERGT M, MOLL R: Urothelial umbrella cells of human ureter are heterogeneous with respect to their uroplakin composition: different degrees of urothelial maturity in ureter and bladder? Eur J Cell Biol 84: 393-405, 2005.

RUBENWOLF PC, GEORGOPOULOS NT, CLEMENTS LA, FEATHER S, HOLLAND P, THOMAS DF, SOUTHGATE J: Expression and localisation of aquaporin water channels in human urothelium in situ and in vitro. Eur Urol 56: 1013-1023, 2009.

RUBENWOLF PC, GEORGOPOULOS NT, KIRKWOOD LA, BAKER SC, SOUTHGATE J: Aquaporin expression contributes to human transurothelial permeability in vitro and is modulated by $\mathrm{NaCl}$. PLoS One 7: e45339, 2012.

SHAFIK A, SIBAI EL O, SHAFIK AA, AHMED I: Do vesical and voided urine have identical compositions? Scand J Urol Nephrol 38: 243-246, 2004.

SHAFIK A, SHAFIK I, SIBAI EL O, SHAFIK AA: Changes in the urine composition during its passage through the ureter. A concept of urothelial function. Urol Res 33: 426-428, 2005.

SHAFIK A, AHMED I, SIBAI EL O, SHAFIK AA: Does the composition of voided urine reflect that of the renal pelvis? Urol Res 34: 261-264, 2006.

SPECTOR DA, WADE JB, DILLOW R, STEPLOCK DA, WEINMAN EJ: Expression, localization, and regulation of aquaporin-1 to -3 in rat urothelia. Am J Physiol Renal Physiol 282: F1034-F1042, 2002.

SPECTOR DA, DENG J, STEWART KJ: Hydration status affects urea transport across rat urothelia. Am J Physiol Renal Physiol 301: F1208-F1217, 2011.

SPECTOR DA, DENG J, STEWART KJ: Hydration status affects sodium, potassium, and chloride transport across rat urothelia. Am J Physiol Renal Physiol. 305: F1669-F1679, 2013.

SPECTOR DA, DENG J, COLEMAN R, WADE JB: The urothelium of a hibernator: the American black bear. Physiol Rep 3: pii: e12429, 2015.

WANG G, LI C, KIM SW, RING T, WEN J, DJURHUUS JC, WANG W, NIELSEN S, FRØKIAER J: Ureter obstruction alters expression of renal acid-base transport proteins in rat kidney. Am J Physiol Renal Physiol 295: F497-F506, 2008.

WILLIAMS NA, BARNARD L, ALLENDER CJ, BOWEN JL, GUMBLETON M, HARRAH T, RAJA A, JOSHI HB: Evidence of nonuniformity in urothelium barrier function between the upper urinary tract and bladder. $J$ Urol 195: 763-770, 2016. 